\title{
ICT based Sustainable Rural Business Opportunities in Developing Countries: A Wireless-Networked RCP-RAP Approach
}

\author{
Md. Abdur Rahman, Mohammad Upal Mahfuz, Kazi M. Ahmed and R.M.A.P. Rajatheva \\ Information and Communications Technologies Program, Telecommunications Program \\ Asian Institute of Technology (AIT), PO Box: 4, Khlongluang, Pathumthani 12120, Thailand
}

\begin{abstract}
Information and Communication Technology (ICT) is gaining much research attention in present times especially for its ability towards poverty alleviation. In this study the prospect of rural business opportunities in developing countries more specifically in the target country, Bangladesh, has been investigated from the viewpoint of wirelessly networked Rural Communication Point (RCP) approach. It is proposed that if only one RCP is set up in every village and there is one Regional Access Point (RAP) responsible for several wirelessly networked RCPs, it is recommended that a significant development is possible in rural areas of the target country with the assistance from existing ICT infrastructure. In this study, the target country is chosen as Bangladesh in South Asia but the similar ICT model can be implemented with success in other countries in the developing world.
\end{abstract}

Key words: ICT, sustainable development, rural business, communication point, information center

\section{INTRODUCTION}

Information and Communication Technology (ICT) has created new opportunities and challenges for developing countries to take full advantage of the potential of ICT to advance their economic and social development. ICT can serve the developing world as a powerful tool to successfully face the on-going process of globalization. Inspired by these opportunities, many developing countries have been aspiring to participate in the global marketplace through e-business, export of software and ICT-enabled services. Over $60 \%$ of the people in the Asia-Pacific region live in rural areas. The rural population tends to be poor, neglected and marginalized groups of society. In comparison to urban areas, the accessibility and availability of Information and Communication Technology (ICT) is significantly lower in rural areas. Equipment support for online connectivity and ICT awareness can greatly contribute to sustainable rural development in the developing countries. On the other hand, ICT can enhance rural productivity. ICT enables solution sharing between local people and communities, providing access to practical information on small business accounting, weather trends and farming based practices, for instance. Timely access to market information via communications networks also helps farmers make astute decisions about what crops to plant and where to sell their products and buy inputs. In Chile, for example, an Internet network among farmer organizations has dramatically increased farmers' incomes by providing information about the crop status, weather, global market prices and training ${ }^{[1]}$. ICT can also provide unprecedented access to rural finance. The financial and information service network can offer micro-finance opportunities for local people and small enterprises.

In this study, Bangladesh has been chosen as the target country as well as a representative of the developing countries in South-Asian region. Bangladesh is a developing country lying in the northeastern part of South-Asia. The economy of the country is largely dependent on agriculture. The farmers cannot sell their products at the appropriate price because they do not get the selling price information of their products at the city centers. As a result, the distributors buy the products from the farmers at lower prices and sell them at the larger cities and thus make the huge profit. For instance, the Internet, far more flexible than the telephone and with email, a far cheaper form of global communications, offers even greater opportunities. The concept of Internet for rural business has already been in use in Africa. For instance, in Kenya, a rural farming cooperative has established a relationship through electronic mail with EarthMarketplace, a US organization to sell local products directly to the American market, bypassing the distributor and increasing the revenues of local farmers ${ }^{[2]}$. In SouthAsian region, an ICT project is being implemented in mountainous and remote areas of northern Pakistan with development in rural business as one of specified project goals ${ }^{[3]}$.

The present ICT infrastructure of Bangladesh: The present ICT infrastructure of Bangladesh has been discussed here. The total number of fixed line telephones operated by Bangladesh Telephone and

Corresponding Author: Md. Abdur Rahman, Information and Communications Technologies Program, Telecommunications Program, Asian Institute of Technology (AIT), PO Box: 4, Khlongluang, Pathumthani 12120, Thailand, Tel: +66 046592590, Fax: +66 (0)2 5245730 
Telegraph Board (BTTB), BRTA and Sheba Telecom is 1 million. Landline telephone networks have covered all 64 districts of the country. There are 4 mobile phone operators serving 3.25 million mobile phone subscribers all over the country. Mobile phones outnumber the number of landlines by $3.25: 1$. Almost $80 \%$ of the populations live in villages, most of whom had previously never used a phone. It is also predicted that the demand of mobile communications will continue to grow strongly, moving at a compounded annual rate of more than $75 \%$ in the near future making Bangladesh a home to 13 million mobile phone users. The total 3.25 million mobile phone users are being served by four network operators named Pacific Bangladesh Telecom CityCell, Grameen Phone, Banglalink and Aktel. Pacific Bangladesh Telecom uses the CDMA system and the other three operators use GSM system for their networks. Starting with only two ISPs and one thousand users in 1996, at present the total number of Internet users in Bangladesh is 2.04 million. The Internet users are being served by 62 Internet Service Providers (ISPs) all over the country. Grameen Telecom's (GTC) Village Phone program has brought the latest communication technologies to rural villages. GTC has made mobile telephony services available to over 35,000 out of Bangladesh's 68,000 villages. Previously detached communities are now connected to the rest of the country and to the world. Urban mobile phones have been made accessible to the rural poor.

Singapore Telecommunications Limited (SingTel) has introduced its broadband IP backbone to Internet Service Providers (ISPs), broadcasters, educational institutions and multinational companies in Bangladesh $^{[4]}$. SingTel efforts can help flourish the ICT sector of Bangladesh through developing faster networking infrastructure in the country. SingTel's broadband IP backbone will operate via satellite in the region. SingTel is providing Digital Video Broadcasting-Internet Protocol (DVB-IP), the network packages IP including voice, video, internet-based applications and other online contents into a DVB stream before transmission via satellite to multiple sites in the Asia Pacific region simultaneously. DVB-IP combines the best of Internet technology with the simultaneous global reach of satellites to deliver instant access to an Internet backbone. Service providers can enjoy significant cost savings on satellite bandwidth compared to the traditional point-to-point technology via the terrestrial network where links have to be dedicated and repeated for each remote site. Furthermore, DVB-IP supports broadband Internet connectivity, video-conference, multimedia video streaming, high-speed file transfer and interactive distance learning and thus it opens up a new horizon for broadcasters to deliver more than just television signals and programming. This technology of the broadband IP backbone via satellite can facilitate the progress of telemedicine in Bangladesh.
Bangladesh is a rural country with some small islands in the southern portion of the country in the Bay of Bengal. The BTTB landline telephone network has not completely reached all the remote corners of the country in part because it is very difficult to draw telephone lines in the soft and marshy island areas with a large number of rivers and canals in between. Since Bangladesh has a small area, dense population and low tele-density, there is a tremendous potential of wireless communications and networking in Bangladesh. The recent abrupt rise in the number of mobile wireless telephone users is an indication for this. Non line of sight (NLOS) wireless technologies is cost-effective options for deploying multi megabit Internet service with ranges from 20 to 50 miles. Line of Sight based previous wireless technologies have proven to be infeasible for use in rural areas due to high installation cost and poor scalability. Mesh NLOS wireless technology may be taken under consideration according to specifically defined site requirements and optimally desired performance ${ }^{[5]}$. Recently, a lot of attention has been observed in the use of wireless networks as a relatively low-cost way of getting access to information database as well as to the Internet. Wi-Fi, packet radio service, microwave links and even $3 \mathrm{G}$ phone networks can be chosen as appropriate wireless networking technologies.

The rural communication point (RCP) approach: In the target country, Bangladesh, for the significant portion of remote and rural land areas, terrestrial communication approaches (such as fiber and microwave links) tend to be very expensive and so apparently infeasible to be established. Satellite systems can be a very good and flexible alternative solution for immediate wide area coverage and broadcast capability. A Rural Communication Point (RCP) can be implemented by applying the combination of a number of leading edge transmission technologies which will enable efficient resource utilization and sharing between many users. In a specific geographic region, a Regional Access Point (RAP) will be responsible for the communication among several wirelessly networked RCPs within that specific region. The RAP will be connected to the satellite and the bandwidth will be distributed among the constituent member RCPs within its coverage area. The RAPs will communicate among themselves via the satellite links. The RCPs can be networked as a Local Area Network (LAN) fashion. One single RCP located in a remote place can also be connected directly to the satellite. These techniques may reduce the satellite bandwidth cost to a significant amount.

\section{THE RCP CONCEPT}

Region-based satellite earth stations: The satellite will connect different region based on broadband networks for data transferring and other 
communications. Each RCP may have a training center and a browsing center. The training center can provide the basic and necessary technological training to the rural people. The browsing center can be used as the Internet café for the community. The café may include a web server to provide web hosting facilities to the general public. The earth station may be used as an ISP to provide Internet service to a limited number of individual users. The wireless LAN can be a good solution for this purpose.

Traffic management: The need of bandwidth and time of service should get some emphasis on the RAP. Some applications (i.e. e-transaction) are very sensitive to time delays while some other requires more bandwidth (i.e. File transfer). Web browsing, which, perhaps, is the most popular Internet service, can tolerate some transmission delays and is also somewhat variable in its required transmission bandwidth. With the combination of factors to consider when sending information, including bit rate, transmission delay and priority, a significant improvement can be gained by appropriate transmission and bandwidth scheduling from a single satellite hub. This traffic shaping part of the RAP concept will work efficiently if located at the traffic concentration point in the earth station. This device will control and monitor the RCP traffic in order to maximize its throughput and to ensure that priorities among different services and traffic types are kept. The traffic will be divided by the service (i.e. Training Centre, Browsing Centre, Individual user) and will be characterized by the time sensitivity factor (video conferencing, web browsing, email etc.).

Efficient transmission technology: Modern modulation and coding systems can give improvements in spectral efficiency (i.e., the amount of transponder bandwidth required for a given bit rate) and the power efficiency (i.e., the transmit power or antenna size required to give the required bit error rate performance). This system can use advanced error correction coding, e.g., Turbo coding with high order QAM modulation and operates to tens of megabits per second and thus can offer a factor of two improvement in either power efficiency or bandwidth efficiency compared to half rate control coding or QPSK modulation. Such a technique will decrease overall system cost by a significant amount.

Last-mile distribution techniques: The RCPs under one RAP will form a LAN to communicate with each other. The medium of the LAN will depend on the terrain and environmental conditions. An efficient distribution technique is required to connect end users to the RCP. Generally, it is assumed that most subscribers will be located within several kilometers from the RCP station and a few may be up to $35 \mathrm{~km}$ away and also that all traffic would be IP-based. While several alternatives, such as ADSL, 3G wireless, LMDS exists, it is believed that modern WLAN can provide an excellent solution for last-mile distribution with speeds up to tens of $\mathrm{Mbps}^{[6]}$. The possible structure of the connections between the RPCs and subscribers are shown in Fig 1.

Adaptive bandwidth allocations to RCPs: In this study the use of conventional bent-pipe transponders are proposed as a cost effective solution for the RAPs. The overall performance of these transponders can be improved by allocating the link capacity depending on the specific RAP requirements. A number of adaptive bandwidth allocation algorithms are available. Various components of the earth station could also be used for video and audio broadcast applications in a costeffective manner. The local redistribution of the broadcast material could then be done from the RCP site, for instance, via UHF transmission.

RCP based approach for poverty alleviation: An RCP based ICT network can help the rural areas develop in many ways. The main problem in developing countries is the urban domination commonly known as 'digital divide' in all technological aspects. Here in this study, a communication model has been proposed that will connect the urban and rural networks without any bound. Free flow of information with all levels will ensure the overall development of the developing country. Figure 2 shows the network structure of the proposed system.

The main users of the system will generally be entrepreneurs, young people, children, women, elderly people (farmers, businessmen and others), unemployed people, NGOs and the Government himself. Most common problems for these users are lack of skills, information, capital, immediate customer base, perceived or actual isolation and poor infrastructure. Figure 3 shows the infrastructure of the information transaction between different networks and communication systems. The RAP will acquire the necessary information from different sources and sort them for different types of end users (i.e. farmers, women, local govt., NGOs etc.) Each community will have their own domain name and web server. Each subscriber will have his own email address. The subscriber will be able to host his own web pages under his own community domain name. The web page will give him an opportunity to introduce his products to the global community. The center may take some steps to design develop software by giving proper training to the young and talented people. Most of the rural women in developing countries know some kinds of handicraft works. The center will introduce their works to the urban and global community where the demand for handicraft is very high. These e-business opportunities will boost up the rural economy by eliminating the middlemen or distributors. 


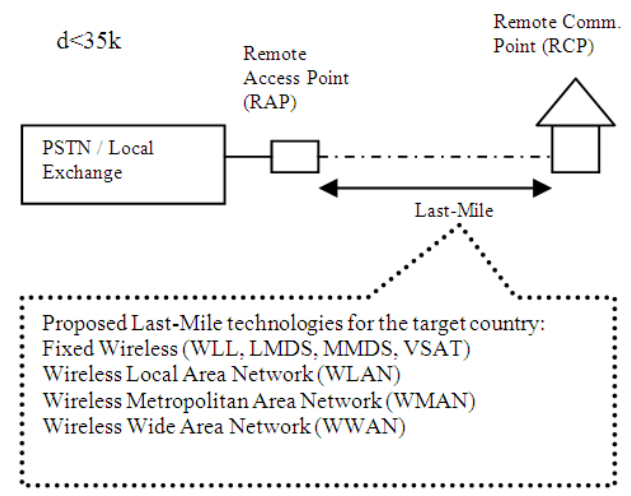

(a)

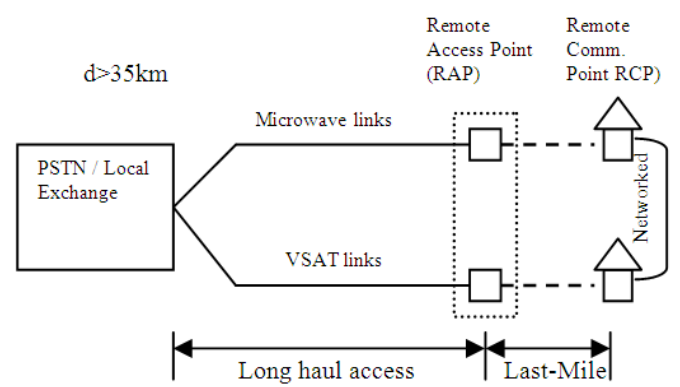

(b)

Fig. 1: Rural access techniques via RCP and RAP in the target country Bangladesh: Distance to PSTN or local exchange (a) $<35 \mathrm{~km}$, (b) $>35 \mathrm{~km}$

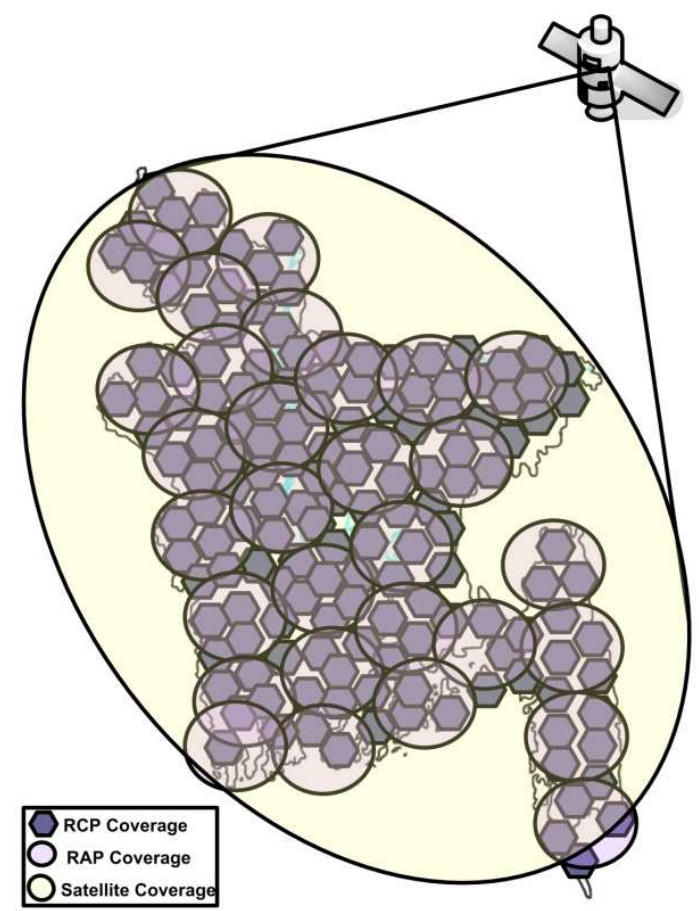

Fig. 2: Rural coverage through RCP, RAP and satellite in the target country Bangladesh

The government banks may give them the capital to initiate their business. Secured transaction of money should be ensured by the information center by using any of the secure online transaction software. In this way, finally the overall services provided by the earth station may be summarized as: Rural office space (phone, fax, copier, printer, computer etc.), Internet service, clerical service and business service, consulting services, forum and training, marketing, networking and shared retail opportunities. All of these services will be provided by the community center. The earth station will charge a small amount of money for these services to make the center self-sustaining and profitable.

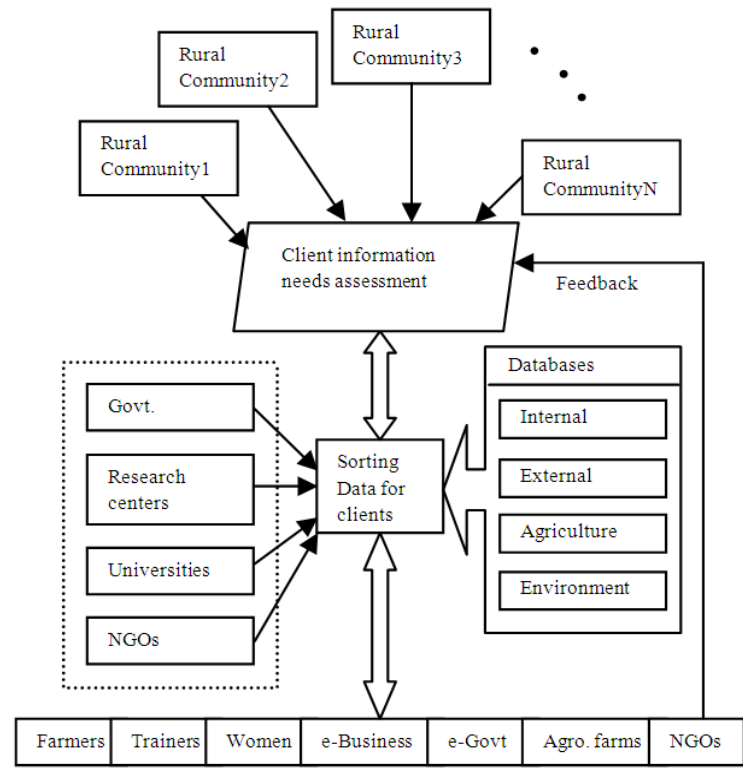

Fig. 3: ICT framework for the proposed RCP based system

The private companies are not generally interested to invest in any infrastructures in rural areas because of poor profit margin. Successful implementation of the rural center will obviously generate some revenue that will attract the private companies to invest there.

Benefits of RCP based rural business opportunities: The benefits of RCP based rural business opportunities are clearly social and financial. A brief discussion of these benefits has been given in the following:

\section{Cost-effectiveness:}

* Turbo coding technology will enhance the system performance and reduce system cost.

* A fixed annual cost per MHz of transponder

* Aggregation of the traffic from all users in one RCP and RAP provides an improvement in the efficiency of the system resources resulting in a further saving compared to individual user access. 


\section{Socio economical benefits:}

* Better communication between rural community and urban as well as international communities. It is also important to view the RCP concept as a way of improving communication within the community itself.

* Better livelihood due to better financial condition arising from ICT based rural business opportunities.

* Promotion of rural tourism World Wide Web design

* Local initiatives which could involve community groups, educational centers and individuals, would also help in promoting indigenous culture, regional communication and improved community awareness.

\section{CONCLUSION}

In this study, we have discussed about the potential use of wireless solutions for providing broadband communications to rural communities. The Rural Communications Points (RCPs) consist of a number of communal institutions, businesses and residential users. Local coordination will play a very important role in this concept. It will be combined with advanced technologies and will efficiently share a single satellite link for a successful implementation. Most of the technology described in this study is currently available. In this study the implementation of ICT based rural business framework and RCP-RAP based technological support have been highly recommended for the developing countries. An appropriate traffic aggregation and management policy with link interface optimization can obviously increase the performance of this proposed concept. It has been shown that in order to function efficiently, the proposed concept requires a community consensus and organization, which would be the driver to the greater communication initiatives within the rural community.

An ICT framework has also been established to incorporate the key elements of a well-functioning and pluralistic rural information and knowledge-based system in the rural areas. The proposed framework is based on the effective use of a variety of ICT applications at the national, regional and local levels combined with supporting institutional development. It is also to be noted that in this study the possibilities of ICT based rural business opportunities for the target country Bangladesh has been discussed only. As the socioeconomic infrastructure of most of the developing countries in the South-Asian region is almost same, a similar approach can be implemented broadly in other developing countries in the region, too. A good number of ICT projects have already received mentionable success in this region. In Sri Lanka the Kothmale Community Radio Internet Project has got a very good success. The project was initiated to test and demonstrate an access model that reduces the barriers and empowers marginalized communities in rural areas, enabling them to benefit from ICTs to expand their knowledge base and thereby support their efforts to tackle their development problems themselves ${ }^{[7]}$. The Government of India has also undertaken a lot of initiatives to empower the rural community. Community Information Centre, Sustainable Access in Rural India, Rural e-seva (West Godavari) etc. The project is worth-mentioning among them. Finally, selfsustainability is a very important issue in the ICT projects in the rural areas of developing countries. In this study, a brief framework of ICT based rural business opportunities has been proposed and the incomes of the ICT center and the rural community itself in broad sense have not been discussed in detail. The income of the ICT center will depend mostly on the community and efficient management. The corresponding Government may give some attention to these centers after successful implementation. The Government offices may use the centers to provide various governmental services to the rural citizens. Such an ICT framework can thus help developing countries achieve a sustainable development in the rural sector.

\section{REFERENCES}

1. Creating a Development Dynamic: ICT for Economic Opportunity. Section 2.2.3, United Nations Development Program, available at http://www.optinit.org/framework/pages/2.2.3.html <http://www.opt-init.org/framework/pages/2.2.3.html> on July 26, 2005.

2. Kenny, C.J., 2000. Expanding Internet access to the rural poor in Africa http://itd.ist.unomaha.edu/Archives/28.pdf. Inform. Technol. Develop. J., 9: 25-31, IOS Press.

3. Hussain, T. and J. Iqbal, 2005. ICT for Rural Development in Mountainous and Remote Areas of Northern Pakistan. Commission on Science and Technology for Sustainable Development in the South (COMSATS), January 2004, web: <http://web.idrc.ca/panasia/ev-51829-201-1-DO_ TOPIC.html> on July 21, 2005.

4. The Daily Star, Feb. 27, 2004. http://www.thedailystar.net/2004/02/27/ on July 26, 2005.

5. Akhter, A.N., Internet Technology in Bangladeshwhere we are and where we are headed. Proc. Am. Assoc. Bangladeshi Engineers and Architects (AABEA) Conf. 2002.

6. Cisco Aironet 1200 Series, Cisco Systems. <http://www.cisco.com/warp/public/cc/pd/witc/ao1 $200 \mathrm{ap} />$ on July 23, 2005.

7. Kothmale Community Radio Internet Project. www1.worldbank.org/publicsector/egov/kothmale_ cs.htm on July 26, 2005. 